\title{
Compressible forced viscous fluid from product Einstein manifolds
}

\author{
Xin $\mathrm{Hao}^{*}$ Bin $\mathrm{Wu}^{\dagger}$ and Liu Zhao \\ School of Physics, Nankai University, Tianjin 300071, China
}

January 19, 2015

\begin{abstract}
We consider the fluctuation modes around a hypersurface $\Sigma_{c}$ in a $(d+2)$ dimensional product Einstein manifold, with $\Sigma_{c}$ taken either near the horizon or at some finite cutoff from the horizon. By mapping the equations that governs the lowest nontrivial order of the fluctuation modes into a system of partial differential equations on a flat Newtonian spacetime, a system of compressible, forced viscous fluid is realized. This result generalizes the non bulk/boundary holographic duality constructed by us recently to the case of a different background geometry.
\end{abstract}

Keywords: Gravity/Fluid correspondence, holography, flat space, compressible fluid

PACS numbers: 04.20.-q, 04.50.Gh

\section{Introduction}

During the last two decades our understanding on the properties of relativistic gravitation has been strengthened considerably. The major new concept which centralizes the studies on gravitation is holographic duality $[1,2]$ originated from the area law of black hole entropy and has been realized in a number of different physical configurations, mostly with gravity in the bulk and some other physical system on the boundary. Among the various realizations of holographic duality, the most important

*email: shanehowe@mail.nankai.edu.cn

${ }^{\dagger}$ email: wubin@mail.nankai.edu.cn

†email: lzhao@nankai.edu.cn, correspondence author. 
ones can be summarized as follows. First comes the well-known AdS/CFT correspondence [3-5], which has led to important applications and remains an active subject of study. Generalization of AdS/CFT has resulted in the so-called AdS/QCD [6,7] and AdS/CMT correspondences. In particular, in the AdS/CMT correspondence, important progresses have been made in the holographic understanding of phase transitions such as superconductivity [8-11], superfluidity [11,12] and so on. Another important aspect of holographic duality is the interpretation of quantum entanglement entropy using a holographic setup $[13,14]$. This line of research seems to be hopeful in interpreting the origin of the area law of the black hole entropy, see [15] for a living review. Besides all these, there is yet another important realization of holographic duality, i.e. the Gravity/Fluid correspondence, see [16-40] for an incomplete list of related works. Unlike other realizations of holographic duality which match the full bulk gravitational degrees of freedom to those of the boundary systems, the Gravity/Fluid correspondence relates only the fluctuation modes of gravity in the bulk to a fluid system on the boundary, and the fluid is usually incompressible. Moreover, the sources of the bulk gravitation contributes to the boundary fluid as an external force, therefore, fluctuation modes of vacuum gravity in the bulk constitute a force-free fluid system on the boundary.

In spite of the numerous success of the theoretical realizations of holographic dual and their applications, a mathematically rigorous definition for holographic duality is still missing. Practically, anything that relates gravity in the bulk to a system on the boundary can be viewed as a realization of holographic duality. In the recent work [40], we have made one further step which breaks the bulk/boundary picture of holographic duality. To be more concrete, what we have realized is a correspondence between fluctuation modes around a vacuum black hole solution with (possibly) curved horizon to a forced, compressible fluid with a stationary density distribution in a flat Newtonian spacetime in one less dimension. In contrast of most well studied cases of Gravity/Fluid correspondences, the fluid system in our work does not live on the boundary of the black hole solution. Moreover, the dual fluid is compressible and subjects to some extra force, although the background gravity solution is source free. This result indicates that holographic duality can be understood beyond the bulk/boundary setting. It is certainly of interests to have more examples of this kind, which may hopefully led to deeper understandings about the essence of holographic duality.

In this work we shall present some further example along the lines of [40]. Instead of a black hole background, we consider fluctuations around a $(d+2)$-dimensional product Einstein manifold $\mathcal{M}=\mathcal{M}_{1} \times \mathcal{M}_{2}$, where $\operatorname{dim}\left(\mathcal{M}_{1}\right)=2$, $\operatorname{dim}\left(\mathcal{M}_{1}\right)=d$. Following a process which is parallel to that made in [40], we will show that similar fluid dual can be realized in a flat Newtonian spacetime with one less dimension. Moreover, we will show that the near horizon limit is not essential in the construction - we can realize similar flat space fluid system by looking at the fluctuation modes around a finite cutoff surface in the background geometry as well. Since the technics used is extremely similar to that of [40], we shall be as brief as possible in the main text and just present the brief route leading to the results. 


\section{Product Einstein manifolds in $(d+2)$-dimensions and hypersurface geometry}

The $(d+2)$ dimensional product Einstein manifold $\mathcal{M}$ which we consider is equipped with the line element

$$
\mathrm{d} s_{\mathcal{M}}^{2}=g_{\mu \nu} \mathrm{d} x^{\mu} \mathrm{d} x^{\nu}=-f(r) \mathrm{d} u^{2}+2 \mathrm{~d} u \mathrm{~d} r+e^{\Phi(x)} \delta_{i j} \mathrm{~d} x^{i} \mathrm{~d} x^{j},
$$

where $(u, r)$ and $\left(x^{i}\right)$ are coordinates on $\mathcal{M}_{1}$ and $\mathcal{M}_{2}$ respectively,

$$
f(r)=1-\omega r-\frac{2 \Lambda}{d} r^{2},
$$

and $\Phi(x)$ obeys the following system of equations:

$$
\begin{aligned}
& \delta^{j k} \partial_{j} \partial_{k} \Phi+\frac{d-2}{2}\left(2 \partial_{i}^{2} \Phi+\delta^{j k} \partial_{j} \Phi \partial_{k} \Phi-\left(\partial_{i} \Phi\right)^{2}\right)+\frac{4}{d} \Lambda e^{\Phi}=0, \\
& \quad \text { (no summation over } i) \\
& (d-2)\left(\partial_{i} \partial_{j} \Phi-\frac{1}{2} \partial_{i} \Phi \partial_{j} \Phi\right)=0 \quad(i \neq j) .
\end{aligned}
$$

For generic $d$, we present a special solution to the equations (3) and (4) in the appendix. More solutions might exist because these equations are nonlinear. Given any solution to eqs. (3) and (4), the metric $g_{\mu \nu}$ of the total manifold $\mathcal{M}$ obeys the vacuum Einstein equation

$$
G_{\mu \nu}=-\Lambda g_{\mu \nu}
$$

We assume that $\omega>0$ and is sufficiently large when $\Lambda<0$, so that $f(r)$ always has zeros. Let the (biggest, if $\Lambda<0$ ) zero of $f(r)$ be $r_{h}$, which represents the radius of a horizon. Consider a $(d+1)$-dimensional timelike hypersurface $\Sigma_{c}$ located at $r=r_{c}$. Evidently, we need $r_{c}>r_{h}$ when $\Lambda<0$ and $r_{c}<r_{h}$ when $\Lambda \geq 0$ in order to ensure that $\Sigma_{c}$ is timelike. The induced line element on this hypersurface is given by

$$
\begin{aligned}
\mathrm{d} s_{\Sigma_{c}}^{2}=\gamma_{a b} \mathrm{~d} x^{a} \mathrm{~d} x^{b} & =-f\left(r_{c}\right) \mathrm{d} u^{2}+e^{\Phi} \delta_{i j} \mathrm{~d} x^{i} \mathrm{~d} x^{j} \\
& =-\left(\mathrm{d} x^{0}\right)^{2}+e^{\Phi} \delta_{i j} \mathrm{~d} x^{i} \mathrm{~d} x^{j} \\
& =-\frac{1}{\lambda^{2}} \mathrm{~d} \tau^{2}+e^{\Phi} \delta_{i j} \mathrm{~d} x^{i} \mathrm{~d} x^{j}
\end{aligned}
$$

where $\tau=\left(\lambda \sqrt{f_{c}}\right) u, x^{0}=\sqrt{f_{c}} u, f_{c}$ is a shorthand for $f\left(r_{c}\right)$. Similar notations have been used in [40]. One can easily promote the hypersurface tensor $\gamma_{a b}$ to a bulk tensor $\gamma_{\mu \nu}$ and define the extrinsic curvature of $\Sigma_{c}$ as $K_{\mu \nu}=\frac{1}{2} \mathscr{L}_{n} \gamma_{\mu \nu}$, with $n^{\mu}$ being a unit vector field which is normal to $\Sigma_{c}$ at $r=r_{c}$. In the coordinates $\left(u, r, x^{i}\right), n^{\mu}$ has components

$$
n^{\mu}=\left(\frac{1}{\sqrt{f}}, \sqrt{f}, 0, \cdots, 0\right)
$$


The projection of (5) on $\Sigma_{c}$ yields the momentum and Hamiltonian constraints,

$$
\begin{aligned}
& D_{a}\left(K_{b}^{a}-\gamma_{b}^{a} K\right)=0, \\
& \hat{R}+K^{a b} K_{a b}-K^{2}=2 \Lambda,
\end{aligned}
$$

where $\hat{R}$ is the Ricci scalar of $\Sigma_{c}, D_{a}$ is the covariant derivative that is compatible with $\gamma_{a b}$. In terms of the Brown-York tensor $t_{a b}=\gamma_{a b} K-K_{a b}$, the momentum and Hamiltonian constraints can be rewritten as

$$
\begin{aligned}
& D_{a} t_{b}^{a}=0, \\
& \hat{R}+t^{a}{ }_{b} t^{b}{ }_{a}-\frac{t^{2}}{d}=2 \Lambda .
\end{aligned}
$$

Explicit values of the extrinsic curvature and the Brown-York tensor will be made use of in the following context, so we present these values by direct calculations,

$$
\begin{array}{ll}
K^{\tau}=\frac{f_{c}^{\prime}}{2 \sqrt{f_{c}}}, & K_{i}^{\tau}=0, \\
K^{i}{ }_{j}=0, & K=K^{a}{ }_{a}=\frac{f_{c}^{\prime}}{2 \sqrt{f_{c}}} .
\end{array}
$$

These in turn lead to the background Brown-York tensor

$$
\begin{aligned}
t_{\tau}^{\tau(B)} & =0, & t^{\tau(B)} & =0, \\
t^{i(B)}{ }_{j} & =\frac{f_{c}^{\prime}}{2 \sqrt{f_{c}}} \delta^{i}{ }_{j}, & t^{(B)} & =t^{a(B)}=\frac{d}{2} \frac{f_{c}^{\prime}}{\sqrt{f_{c}}} .
\end{aligned}
$$

We have intentionally added a superscript $(B)$ to indicate that these are the values with respect to the background geometry. When considering fluctuation modes, these values must be supplemented with fluctuation modifications.

\section{Petrov I boundary condition and near horizon limit}

In this section we shall consider the case with $\Sigma_{c}$ placed very closed to the horizon, i.e. $r_{c}-r_{h}=\epsilon \alpha^{2} \lambda^{2}$, where $\epsilon=1$ if $\Lambda<0, \epsilon=-1$ if $\Lambda \geq 0$, and $\lambda \rightarrow 0$ is the same parameter appeared in (6). The positive constant $\alpha$ is introduced to eliminate some un-necessary complexity when taking the near horizon limit. We will focus ourselves on the fluctuation modes around $\Sigma_{c}$ and pay particular attention to the Petrov I boundary condition

$$
\left.C_{(l) i(l) j}\right|_{\Sigma_{c}}=\left.l^{\mu}\left(m_{i}\right)^{\nu} l^{\rho}\left(m_{j}\right)^{\sigma} C_{\mu \nu \rho \sigma}\right|_{\Sigma_{c}}=0,
$$

where $C_{\mu \nu \rho \sigma}$ represents the bulk Weyl tensor, and

$$
l^{\mu}=\frac{1}{\sqrt{2}}\left(\frac{1}{\sqrt{f}}\left(\partial_{u}\right)^{\mu}-n^{\mu}\right), \quad k^{\mu}=\frac{1}{\sqrt{2}}\left(\frac{1}{\sqrt{f}}\left(\partial_{u}\right)^{\mu}+n^{\mu}\right),
$$




$$
\left(m_{i}\right)^{\mu}=e^{-\frac{1}{2} \Phi}\left(\partial_{i}\right)^{\mu}
$$

constitute a set of Newman-Penrose basis vector field which obeys

$$
l^{2}=k^{2}=0,,(k, l)=1,,\left(l, m_{i}\right)=\left(k, m_{i}\right)=0,,\left(m_{i}, m_{j}\right)=\delta_{i j} .
$$

When restricted on $\Sigma_{c}$, we can write

$$
\left.l^{\mu}\right|_{\Sigma_{c}}=\frac{1}{\sqrt{2}}\left(\left(\partial_{0}\right)^{\mu}-n^{\mu}\right),\left.\quad k^{\mu}\right|_{\Sigma_{c}}=\frac{1}{\sqrt{2}}\left(\left(\partial_{0}\right)^{\mu}+n^{\mu}\right) .
$$

Therefore the boundary condition can also be cast in the form

$$
C_{0 i 0 j}+C_{0 i j(n)}+C_{0 j i(n)}+C_{i(n) j(n)}=0,
$$

with

$$
\begin{aligned}
& C_{a b c d}=\gamma_{a}^{\mu} \gamma_{b}^{\nu} \gamma_{c}^{\sigma} \gamma_{d}^{\rho} C_{\mu \nu \sigma \rho}=\hat{R}_{a b c d}+K_{a d} K_{b c}-K_{a c} K_{b d}-\frac{4 \Lambda}{d(d+1)} \gamma_{a[c} \gamma_{d] b}, \\
& C_{a b c(n)}=\gamma_{a}^{\mu} \gamma_{b}^{\nu} \gamma_{c}^{\sigma} n^{\rho} C_{\mu \nu \sigma \rho}=D_{a} K_{b c}-D_{b} K_{a c}, \\
& C_{a(n) b(n)}=\gamma_{a}^{\mu} n^{\nu} \gamma_{c}^{\sigma} n^{\rho} C_{\mu \nu \sigma \rho}=-\hat{R}_{a b}+K K_{a b}-K_{a c} K_{b}^{c}+\frac{2 \Lambda}{(d+1)} \gamma_{a b},
\end{aligned}
$$

where $\hat{R}_{a b c d}$ and $\hat{R}_{a b}$ are the Riemann and Ricci tensors of $\Sigma_{c}$. Inserting (18) into (17) and inverting the relationship between $K_{a b}$ and $t_{a b}$, the boundary condition (17) becomes

$$
\begin{gathered}
\frac{2}{\lambda^{2}} t_{i}^{\tau} t^{\tau}{ }_{j}+\frac{t^{2}}{d^{2}} \gamma_{i j}+\frac{2 \Lambda}{d} \gamma_{i j}-\left(t^{\tau}{ }_{\tau}-2 \lambda D_{\tau}\right)\left(\frac{t}{d} \gamma_{i j}-t_{i j}\right) \\
-\frac{2}{\lambda} D_{(i} t^{\tau}{ }_{j)}-t_{i k} t^{k}{ }_{j}-\hat{R}_{i j}=0 .
\end{gathered}
$$

Note that the appearance of $\lambda$ in this equation comes purely from the rescaling of the coordinate $x^{0} \rightarrow \tau / \lambda$.

To see the effects of the fluctuation modes let us expand the metric, Ricci tensor and the Brown-York tensor on $\Sigma_{c}$ in the following form,

$$
\begin{aligned}
& \gamma_{a b}=\gamma_{a b}^{(B)}+\sum_{n=1}^{\infty} \gamma_{a b}^{(n)} \lambda^{n}, \\
& \hat{R}_{a b}=\hat{R}_{a b}^{(B)}+\sum_{n=1}^{\infty} \lambda^{n} \hat{R}_{a b}^{(n)}, \\
& t_{b}^{a}=t_{b}^{a(B)}+\sum_{n=1}^{\infty} \lambda^{n} t_{b}^{a(n)},
\end{aligned}
$$

where the leading terms on the right hand side represent the background values. In the near horizon limit, $f_{c}$ can be rearranged in the form

$$
f_{c}=f_{h}^{\prime} \cdot\left(\epsilon \alpha^{2} \lambda^{2}\right)+\frac{1}{2} f_{h}^{\prime \prime} \cdot\left(\alpha^{2} \lambda^{2}\right)^{2},
$$




$$
f_{h}^{\prime}=-\left(\omega+\frac{4 \Lambda r_{h}}{d}\right), \quad f_{h}^{\prime \prime}=-\frac{4 \Lambda}{d}
$$

which follows from the Taylor series expansion around $r_{h}$. Consequently, the background values given in (12) will also develop $\lambda$ dependences. In the end, we have

$$
\begin{aligned}
& t^{\tau}{ }_{\tau}=0+\lambda t_{\tau}^{\tau(1)}+\cdots, \\
& t^{\tau}{ }_{i}=0+\lambda t_{i}^{\tau(1)}+\cdots, \\
& t^{i}{ }_{j}=\frac{\epsilon}{2}\left(\frac{\sqrt{\epsilon f_{h}^{\prime}}}{\alpha \lambda}-\alpha \lambda \frac{4 \Lambda}{d \sqrt{\epsilon f_{h}^{\prime}}}\right) \delta^{i}{ }_{j}+\lambda t^{i(1)}+\cdots, \\
& t=\frac{d \epsilon}{2}\left(\frac{\sqrt{\epsilon f_{h}^{\prime}}}{\alpha \lambda}-\alpha \lambda \frac{4 \Lambda}{d \sqrt{\epsilon f_{h}^{\prime}}}\right)+\lambda t^{(1)}+\cdots .
\end{aligned}
$$

What we need to do is to expand (9), (10) and (19) into power series in $\lambda$ and look at the first nontrivial order contributions. For this purpose we need to supplement (23) with expansions of $\hat{R}_{a b}$ and $D_{(i} t^{\tau}{ }_{j)}$. To evaluate the latter we need to expand the Christoffel connection $\hat{\Gamma}_{b c}^{a}$. Omitting the details we present the results as follows:

$$
\begin{gathered}
\hat{\Gamma}_{b c}^{a}=\Gamma_{b c}^{a(B)}+\mathcal{O}\left(\lambda^{1}\right), \\
\hat{R}_{a b}=\hat{R}_{a b}^{(B)}+\mathcal{O}\left(\lambda^{1}\right),
\end{gathered}
$$

where the background values

$$
\begin{array}{ll}
\hat{\Gamma}_{a b}^{\tau(B)}=\hat{\Gamma}_{\tau b}^{a(B)}=0, & \hat{\Gamma}_{i j}^{k(B)}=\frac{1}{2}\left(\delta^{k}{ }_{i} \partial_{j} \Phi+\delta^{k}{ }_{j} \partial_{i} \Phi-\delta_{i j} \partial^{k} \Phi\right), \\
\hat{R}_{\tau a}^{(B)}=0, & \hat{R}_{i j}^{(B)}=\frac{2 \Lambda}{d} e^{\Phi} \delta_{i j}
\end{array}
$$

are all $\lambda$-independent. Therefore, we have

$$
\begin{aligned}
D_{(k} t^{\tau}{ }_{j)} & =\partial_{(k}\left(\lambda t_{j)}^{\tau(1)}+\mathcal{O}\left(\lambda^{2}\right)\right)-\left(\hat{\Gamma}_{(k j)}^{l(B)}+\mathcal{O}\left(\lambda^{1}\right)\right)\left(\lambda t_{l}^{\tau(1)}+\mathcal{O}\left(\lambda^{2}\right)\right) \\
& \equiv \lambda \zeta_{k j}^{(1)}+\lambda^{2} \zeta_{k j}^{(2)}+\mathcal{O}\left(\lambda^{3}\right),
\end{aligned}
$$

where

$$
\zeta_{k j}^{(1)}=\partial_{(k} t_{j)}^{\tau(1)}-\partial_{(k} \Phi t_{j)}^{\tau(1)}+\frac{1}{2} \delta_{k j} \delta^{l m} \partial_{l} \Phi t_{m}^{\tau(1)}
$$

and $\zeta_{k j}^{(2)}$ is some complicated expression which depends on $\gamma_{k j}^{(1)}$ and $t_{j}^{\tau(2)}$. We do not need to use the explicit form of $\zeta_{k j}^{(2)}$ in this paper.

Substituting eqs. (23) and (25) into (19), we get in the first nontrivial order $\mathcal{O}\left(\lambda^{0}\right)$ the following identity,

$$
t_{j}^{i(1)}=\frac{\alpha \epsilon}{\sqrt{\epsilon f_{h}^{\prime}}} \cdot 2 \gamma^{i k(0)}\left(t_{k}^{\tau(1)} t_{j}^{\tau(1)}-\zeta_{k j}^{(1)}\right)+\frac{1}{d} t^{(1)} \delta_{j}^{i},
$$


where $\gamma^{i k(0)}=e^{-\Phi} \delta^{i k}$. Notice that the dependences on $\alpha$ and $\epsilon$ can be get rid of by choosing $\alpha=\epsilon \sqrt{\epsilon f_{c}^{\prime}}$, which we will take from now on. Similarly, expanding the $\tau$ component of (9) into power series in $\lambda$ yields, at order $\mathcal{O}\left(\lambda^{-1}\right)$,

$$
\delta^{i j}\left(\partial_{i}+\frac{d-2}{2} \partial_{i} \Phi\right) t_{j}^{\tau(1)}=0
$$

and expanding the spatial components of the same equation yields, at the order $\lambda^{1}$, the following equation,

$$
\partial_{\tau} t_{i}^{\tau(1)}-\frac{1}{2}\left(t^{(1)}-t_{\tau}^{\tau(1)}\right) \partial_{i} \Phi+\left(\partial_{j}+\frac{d}{2} \partial_{j} \Phi\right) t_{i}^{j(1)}=0
$$

The lowest order contribution to the Hamiltonian constraint is at order $\mathcal{O}\left(\lambda^{0}\right)$, which yields

$$
t_{\tau}^{\tau(1)}=-2 \gamma^{i j(0)} t_{i}^{\tau(1)} t_{j}^{\tau(1)}
$$

The form of the equations (27), (28), (29) and (30) is exactly the same as those appeared in [40] - though with different values for each quantity entering the equations - which had led to a flat space fluid system after introducing some appropriate holographic dictionary. Therefore, we can follow the same line of argument as made in [40] and introduce the holographic dictionary

$$
\rho=e^{\frac{d}{2} \Phi}, \quad \mu=e^{\frac{d-2}{2} \Phi}, \quad \nu=\frac{\mu}{\rho}=e^{-\Phi},
$$

and

$$
t_{i}^{\tau(1)}=\frac{v_{i}}{2 \nu}, \quad \frac{t^{(1)}}{d}=\frac{p}{2 \mu},
$$

where $\rho, \mu, v_{i}, p$ are to be interpreted as the density, viscosity, velocity field and the pressure of the dual fluid. Finally, eq. (28) becomes the continuity equation

$$
\partial^{j}\left(\rho v_{j}\right)=0
$$

and eqs. (27) and (29) combined together give rise to the standard equation

$$
\rho\left(\partial_{\tau} v_{i}+v^{j} \partial_{j} v_{i}\right)=-\partial_{i} p+\partial^{j} d_{i j}+f_{i}
$$

for the velocity field of the fluid, where

$$
d_{i j}=\mu\left(\partial_{j} v_{i}+\partial_{i} v_{j}-\frac{2}{d} \delta_{i j} \partial^{k} v_{k}\right)
$$

represents the deviatoric stress, which is symmetric traceless and depends only on the derivatives of the velocity field, hence vanishes in the hydrostatic equilibrium limit, and

$$
f_{i}=\partial^{j} \Phi\left(d_{i j}+\frac{d-2}{2} p \delta_{i j}\right)+\frac{2}{d} v^{j} v_{j} \partial_{i} \rho-\frac{2}{d}\left(v^{j} \partial_{j} \rho\right) v_{i}
$$


represents an extra force. Since the first two terms $-\partial_{i} p+\partial^{j} d_{i j}$ on the right hand side of (34) correspond to the ordinary surface forces, the last term which cannot be cast in the form of the first two terms must represent a body force. The equations (33) and (34) constitute a system of equations governing the motion of a compressible, forced, stationary and viscous fluid moving in the $(d+1)$-dimensional Newtonian spacetime $\mathbb{R} \times \mathbb{E}^{d}$.

\section{Finite cutoff}

The whole construction of the last section is very similar to that made in [40] for black hole background. Now we would like to ask a different question: Can we construct a flat space fluid dual without making use of the near horizon Petrov I boundary condition?

To answer this question, let us go over the whole process of the construction. It is clear the every formulae until (22) still holds if $\Sigma_{c}$ is not placed near the horizon. However, from eq. (23) and onwards, things begin to change a little. Concretely, (23) must be replaced by

$$
\begin{aligned}
& t^{\tau}{ }_{\tau}=0+\lambda t_{\tau}^{\tau(1)}+\cdots, \\
& t^{\tau}{ }_{i}=0+\lambda t^{\tau(1)}+\cdots, \\
& t^{i}{ }_{j}=\frac{f_{c}^{\prime}}{2 \sqrt{f_{c}}} \delta^{i}{ }_{j}+\lambda t^{i(1)}+\cdots, \\
& t=\frac{d}{2} \frac{f_{c}^{\prime}}{\sqrt{f_{c}}}+\lambda t^{(1)}+\cdots,
\end{aligned}
$$

where $f_{c}, f_{c}^{\prime}$ etc must be kept un-expanded. If we replace (23) with (37) and carry on the rest process as made in the last section, then we will encounter some trouble. Unlike what has been derived in the near horizon limit, at finite cutoff the first nontrivial order of the boundary condition becomes

$$
t_{k}^{\tau(1)} t_{j}^{\tau(1)}-\zeta_{k j}^{(1)}=0
$$

insert (38) into (28), we could only come to the conclusion that at order $\mathcal{O}\left(\lambda^{1}\right)$ the momentum constraints will not allow fluctuation in the $t_{i}^{\tau}$ component, i.e.

$$
t_{i}^{\tau(1)}=0
$$

If we expand the boundary condition to the next order, then the following relation would follow,

$$
t_{j}^{i(1)}=\frac{t^{(1)}}{d} \delta_{j}^{i}-\frac{\sqrt{f_{c}}}{f_{c}^{\prime}}\left(R_{j}^{i(1)}+2 \zeta_{j}^{i(2)}\right),
$$

where

$$
\zeta_{j}^{i(2)}=\gamma^{i k(1)} \zeta_{k j}^{(1)}+\gamma^{i k(0)} \zeta_{k j}^{(2)}
$$


Using (40) we can the spatial component of the momentum constraints (29) into the following equation:

$$
\begin{aligned}
\frac{1}{d} \partial_{i} t^{(1)} & =\frac{\sqrt{f_{c}}}{f_{c}^{\prime}}\left(\partial_{j}+\frac{d}{2} \partial_{j} \Phi\right)\left(R_{i}^{j(1)}+2 \zeta_{i}^{j(2)}\right) \\
& =\frac{\sqrt{f_{c}}}{f_{c}^{\prime}} e^{-\frac{d}{2} \Phi} \partial_{j}\left[e^{\frac{d}{2} \Phi}\left(R_{i}^{j(1)}+2 \zeta_{i}^{j(2)}\right)\right]
\end{aligned}
$$

but it is exceedingly hard to explain (41) as a partial differential equation that describes the fluid motion.

To avoid the above problem and realize the fluid dual at finite cutoff, we employ a translation of $\Phi$ as follows:

$$
\Phi \rightarrow \Phi-\ln \lambda
$$

Such a translation does not alter the order of derivatives of $\Phi$ but does change the order of $e^{\Phi}$. After the translation (42), the spatial components of the induced metric (6) will be rescaled:

$$
\gamma_{a b}^{(B)} \mathrm{d} x^{a} \mathrm{~d} x^{b} \rightarrow \tilde{\gamma}_{a b}^{(B)} \mathrm{d} x^{a} \mathrm{~d} x^{b}=-\frac{1}{\lambda^{2}} \mathrm{~d} \tau^{2}+\frac{e^{\Phi}}{\lambda} \delta_{i j} \mathrm{~d} x^{i} \mathrm{~d} x^{j}
$$

and eq. (3) becomes

$$
\delta^{j k} \partial_{j} \partial_{k} \Phi+\frac{d-2}{2}\left(2 \partial_{i}^{2} \Phi+\delta^{j k} \partial_{j} \Phi \partial_{k} \Phi-\left(\partial_{i} \Phi\right)^{2}\right)+\frac{4 \Lambda}{d} \frac{e^{\Phi}}{\lambda}=0 .
$$

As a result the Ricci tensor of $\Sigma_{c}$ will be inversely proportional to $\lambda$,

$$
\hat{R}_{i j}^{(B)} \rightarrow \tilde{R}_{i j}^{(B)}=\frac{2 \Lambda}{d} \frac{e^{\Phi}}{\lambda} \delta_{i j}
$$

The background Brown-York tensor $t_{b}^{a(B)}$, the Christoffel connection $\hat{\Gamma}_{b c}^{a(B)}$ and $\zeta_{i j}^{(1)}$ are all kept invariant. So we can list the new boundary condition as

$$
\begin{gathered}
\frac{2}{\lambda^{2}} \tilde{\gamma}^{i k} t^{\tau}{ }_{k} t^{\tau}{ }_{j}+\frac{t^{2}}{d^{2}} \delta^{i}{ }_{j}+\frac{2 \Lambda}{d} \delta^{i}{ }_{j}-\left(t^{\tau}{ }_{\tau}-2 \lambda D_{\tau}\right)\left(\frac{t}{d} \delta^{i}{ }_{j}-t^{i}{ }_{j}\right) \\
-\frac{2}{\lambda} \tilde{\gamma}^{i k} D_{(k} t^{\tau}{ }_{j)}-t^{i}{ }_{k} t^{k}{ }_{j}-\hat{R}^{i}{ }_{j}=0 .
\end{gathered}
$$

The form of this equation looks identical to (19), however with a different background metric $\tilde{\gamma}^{i j}$ which is $\lambda$ dependent. Using (46) in place of (19) and inserting (25) and (37), we get the following new equation at the order $\mathcal{O}\left(\lambda^{1}\right)$,

$$
t_{j}^{i(1)}=\frac{\sqrt{f_{c}}}{f_{c}^{\prime}} \cdot 2 \gamma^{i k(0)}\left(t_{k}^{\tau(1)} t^{\tau(1)}-\zeta_{k j}^{(1)}\right)+\frac{1}{d} t^{(1)} \delta^{i}{ }_{j}-\frac{\sqrt{f_{c}}}{f_{c}^{\prime}} \hat{R}_{j}^{i(1)} .
$$


Obviously the momentum constraints are invariant under the translation (42) for $\Phi$, so there will not be any new terms in equation (28) and (29). Substituting (47) into (29), and introducing the following holographic dictionary

$$
\begin{array}{ll}
\rho=\frac{f_{c}^{\prime}}{\sqrt{f_{c}}} e^{\frac{d}{2} \Phi}, & \mu=e^{\frac{d-2}{2} \Phi}, \quad \nu=\frac{\mu}{\rho}=\frac{\sqrt{f_{c}}}{f_{c}^{\prime}} e^{-\Phi}, \\
t_{i}^{\tau(1)}=\frac{v_{i}}{2 \nu}, & \frac{t^{(1)}}{d}=\frac{p}{2 \mu},
\end{array}
$$

eqs. (28) and (29) will again become the continuity equation and the Navier-Stokes equation

$$
\begin{aligned}
& \partial^{j}\left(\rho v_{j}\right)=0, \\
& \rho\left(\partial_{\tau} v_{i}+v^{j} \partial_{j} v_{i}\right)=-\partial_{i} p+\partial^{j} d_{i j}+f_{i},
\end{aligned}
$$

where $d_{i j}$ takes the same form as in (35) (of course with different $\mu$ and $v_{i}$ ), and

$$
f_{i}=\partial^{j} \Phi\left(d_{i j}+\frac{d-2}{2} p \delta_{i j}\right)+\frac{2}{d} v^{j} v_{j} \partial_{i} \rho-\frac{2}{d}\left(v^{j} \partial_{j} \rho\right) v_{i}+\frac{2 \sqrt{f_{c}}}{f_{c}^{\prime}} \nu \partial_{j}\left(\rho \hat{R}_{i}^{j(1)}\right) .
$$

Comparing to the case of the last section, we see that an extra force term $\frac{2 \sqrt{f_{c}}}{f_{c}^{\prime}} \nu \partial_{j}\left(\rho \hat{R}_{i}^{j(1)}\right)$ appears in $f_{i}$ at finite cutoff.

\section{Concluding remarks}

The result of this work indicates that a compressible, forced, viscous fluid in flat Newtonian spacetime can not only be realized as the holographic dual of fluctuation modes around a black hole background, but also be realized as the dual of fluctuating modes around a product Einstein manifold. The construction relies on taking a timelike hypersurface $\Sigma_{c}$ - which is placed either near the horizon or at some finite cutoff - and mapping it into an Euclidean space which lies in its conformal class. It is surprising that the final fluid equations, including the form of the extra force term $f_{i}$, are basically the same as that arising from fluctuations around a black hole background (though in the case of a finite cutoff a novel force term appears). It remains to understand the nature of the extra force term from the point of view of fluid dynamics.

Experienced readers on Gravity/Fluid correspondence might feel that the fluid equations in flat space are only those for a curved space incompressible fluid in disguise. This is true in some sense. However, writing the fluid equations in flat Newtonian spacetime is still a nontrivial step forward, because the holographic dictionary we adopt is quite different for that leading to a curved space incompressible fluid. In practice, any physical system which is dual to another one in some way can be regarded as its dual in disguise. However this view point does not disvalue the duality relationship between the two systems. 
As a technical addendum let us mention why we stick to the approach which makes use of the Petrov I boundary condition rather than adopting the boost-rescaling approach which is also widely used in Gravity/Fluid correspondence [18-29]. The reason lies in that the background geometry which we use is not necessarily flat, and boosting requires the existence of a flat boundary. For this reason, we do not expect the boost-rescaling approach to be applicable in our situations.

The product manifold taken in this work is of the simplest kind, i.e. the total manifold is the product of two submanifolds. We can of course consider the case when the total manifold is the product of several submanifolds, e.g. the submanifold $\mathcal{M}_{2}$ itself has a product structure, with line element of the form

$$
\mathrm{d} s_{\mathcal{M}_{2}}^{2}=e^{\Phi(x)} \delta_{i j} \mathrm{~d} x^{i} \mathrm{~d} x^{j}+e^{\Psi(y)} \delta_{m n} \mathrm{~d} y^{m} \mathrm{~d} y^{n}+\cdots
$$

We expect that flat space fluid may still be constructed out of the fluctuating modes around such backgrounds, and the resulting fluid might become anisotropic. We will have more to say along the lines of research of [40] and the present work.

\section{Appendix}

In this appendix we discuss some properties regarding the system of equations (3) and (4) that determine the function $\Phi(x)$.

For the particular case of $d=2$, these equations degenerate into a single equation

$$
\delta^{j k} \partial_{j} \partial_{k} \Phi+2 \Lambda e^{\Phi}=0
$$

which can be easily recognized to be the Euclideanized Liouville equation for $\Lambda \neq 0$ or the Laplacian equation for $\Lambda=0$. The fact that the metric function $\Phi$ obeys Liouville or Laplacian equation in the case $d=2$ is first observed in [41] while considering black hole metrics. In the case of product Einstein manifold such equations still hold. Existence of infinitely many solutions to such equations are well known.

For $d>2$, these equations are much more complicated. However, there is an explicit solution which we reproduce below. Let

$$
\Phi=-2 \log \chi
$$

Then, eq. (4) becomes

$$
\partial_{i} \partial_{j} \chi=0, \quad i \neq j .
$$

Therefore, $\chi$ must take the form

$$
\chi=\sum_{i} \chi_{i}\left(x^{i}\right),
$$


where each term depends on a single $x^{i}$. Moreover, eq. (3) can be rewritten as

$$
\sum_{k} \frac{(d-1)\left(\partial_{k} \chi\right)^{2}-\chi \partial_{k}^{2} \chi}{\chi^{2}}-(d-2) \frac{\chi \partial_{i}^{2} \chi}{\chi^{2}}+\frac{4 \Lambda}{d \chi^{2}}=0 .
$$

Suppose

$$
\chi=a+b \sum_{i} x_{i}^{2}
$$

eq. (53) becomes an algebraic equation

$$
\frac{\Lambda}{d}-a b(d-1)=0
$$

for the coefficients $a$ and $b$. When $\Lambda \neq 0$, both $a$ and $b$ cannot vanish. So, we can freely choose $a=1$ and then get

$$
b=\frac{\Lambda}{d(d-1)}
$$

This yields the solution

$$
\chi(x)=1+\frac{\Lambda}{d(d-1)} \sum_{k} x_{k}^{2}
$$

i.e.

$$
e^{\Phi}=\frac{1}{\chi^{2}}=\frac{1}{\left(1+\frac{\Lambda}{d(d-1)} \sum_{k} x_{k}^{2}\right)^{2}}
$$

If $\Lambda=0$, then either $a$ or $b$ must be zero. Taking $a=0$ and let $b$ be arbitrary, we get

$$
\chi(x)=b \sum_{k} x_{k}^{2}
$$

i.e.

$$
e^{\Phi}=\frac{1}{\chi^{2}}=\frac{1}{b^{2}\left(\sum_{k} x_{k}^{2}\right)^{2}} .
$$

$e^{\Phi}$ becomes singular in this case at $x_{i}=0$. Alternatively, we can take $b=0$ and normalize $a=1$, which corresponds to $\chi=1$, i.e. $\Phi=0$. In this particular case the fluid we obtain becomes incompressible but still lives in flat space, and the force $f_{i}$ given in (36) (and also the first two terms in (51)) becomes vanishing. Since eqs. (3) and (4) are essentially nonlinear, there may be other solutions to these equations.

\section{References}

[1] G. 't Hooft, "Dimensional reduction in quantum gravity", Salamfest 1993:0284-296, [arXiv:gr-qc/9310026]. 
[2] L. Susskind, "The World as a Hologram", J. Math. Phys. 36 (1995) 6377-6396, [arXiv:hep-th/9409089].

[3] J. M. Maldacena, "The large $N$ limit of superconformal field theory and supergravity", Adv. Theor. Math. Phys. 2, 231 (1998) [arXiv:hep-th/9711200].

[4] S. S. Gubser, I. R. Klebanov, A. M. Polyakov, "Gauge theory correlators from noncritical string theory", Phys. Lett. B 428, 105 (1998) [arXiv:hep-th/9802109].

[5] E. Witten, "Anti de Sitter space and holography", Adv. Theor. Math. Phys. 2, 253 (1998) [arXiv:hep-th/9802150].

[6] P. Kovtun, D. T. Son, and A. O. Starinets, "Holography and hydrodynamics: diffusion on stretched horizons", JHEP 0310:064 (2003) [arXiv:hep-th/0309213].

[7] S. J. Brodsky, G. F. de Téramond, and A. Deur, "The AdS/QCD Correspondence and Exclusive Processes", [arXiv:1007.5385].

[8] S. A. Hartnoll, C. P. Herzog, and G. T. Horowitz, "Building an AdS/CFT superconductor", Phys.Rev.Lett.101:031601 (2008) [arXiv:0803.3295].

[9] S. A. Hartnoll, C. P. Herzog, and G. T. Horowitz, "Holographic Superconductors", JHEP 0812:015 (2008) [arXiv:0810.1563].

[10] G. T. Horowitz, "Introduction to Holographic Superconductors", [arXiv:1002.1722].

[11] C. P. Herzog, "Lectures on Holographic Superfluidity and Superconductivity", J.Phys.A42:343001 (2009) [arXiv:0904.1975].

[12] C. P. Herzog, P. K. Kovtun, and D. T. Son, "Holographic model of superfluidity", Phys.Rev. D79 (2009) 066002 [arXiv:0809.4870].

[13] T. Nishioka, S. Ryu, and T. Takayanagi, "Holographic Entanglement Entropy: An Overview", J.Phys.A42:504008 (2009) [arXiv:0905.0932].

[14] T. Takayanagi, "Entanglement Entropy from a Holographic Viewpoint", Class.Quant.Grav. 29 (2012) 153001 [arXiv:1204.2450v2].

[15] S. N. Solodukhin, "Entanglement entropy of black holes", Living Rev. Relativity 14, (2011), 8 [arXiv:1104.3712v1].

[16] I. Bredberg, C. Keeler, V. Lysov and A. Strominger, "Wilsonian Approach to Fluid/Gravity Duality", JHEP 1103, 141 (2011) [arXiv:1006.1902].

[17] I. Bredberg, C. Keeler, V. Lysov and A. Strominger, "From Navier-Stokes to Einstein", JHEP 1207, 146 (2012) [arXiv:1101.2451].

[18] G. Compere, P. McFadden, K. Skenderis, M. Taylor, "The holographic fluid dual to vacuum Einstein gravity", JHEP 07, 050 (2011) [arXiv:1103.3022].

[19] R. G. Cai, L. Li and Y. L. Zhang, "Non-Relativistic fluid dual to asymptotically AdS gravity at finite cutoff surface", JHEP 1107:027 (2011) [arXiv:1104.3281].

[20] C. Eling, A. Meyer, Y. Oz, "The relativistic Rindler hydrodynamics", JHEP 1205, 116 (2012) arXiv:1201.2705.

[21] G. Compere, P. McFadden, K. Skenderis and M. Taylor, "The relativistic fluid dual to vacuum Einstein gravity", JHEP 1203, 076 (2012) [arXiv:1201.2678].

[22] G. Chirco, C. Eling and S. Liberati, "Higher Curvature Gravity and the Holographic fluid dual to flat spacetime", JHEP 1108, 009 (2011) [arXiv:1105.4482].

[23] X. J. Bai, Y. P. Hu, B. H. Lee and Y. L. Zhang, "Holographic Charged Fluid with Anomalous Current at Finite Cutoff Surface in Einstein-Maxwell Gravity", JHEP 1211, 054 (2012) [arXiv:1207.5309]. 
[24] D. C. Zou, S. J. Zhang and B. Wang, "Holographic charged fluid dual to third order Lovelock gravity", Phys. Rev. D 87, 084032 (2013) [arXiv:1302.0904].

[25] Y. P. Hu and J. H. Zhang, "Gravity/Fluid Correspondence and Its Application on Bulk Gravity with $U$ (1) Gauge Field", Adv. High Energy Phys. 2014, 483814 (2014) [arXiv:1311.3974].

[26] C. Niu, Y. Tian, X. N. Wu and Y. Ling, "Incompressible Navier-Stokes Equation from EinsteinMaxwell and Gauss-Bonnet-Maxwell Theories", Phys. Lett. B 711, 411 (2012) [arXiv:1107.1430].

[27] R. G. Cai, L. Li, Z. Y. Nie and Y. L. Zhang, "Holographic forced fluid dynamics in non-relativistic limit", Nucl. Phys. B 864, 260 (2012) [arXiv:1202.4091].

[28] R. G. Cai, T. J. Li, Y. H. Qi and Y. L. Zhang, "Incompressible Navier-Stokes Equations from Einstein Gravity with Chern-Simons Term", Phys. Rev. D 86, 086008 (2012) [arXiv:1208.0658].

[29] Y. Tian, X. N. Wu, H. B. Zhang, "Poor man's holography: How far can it go?", Class. Quant. Grav. 30, 125010 (2013) [arXiv:1204.2029].

[30] V. Lysov, and A. Strominger, "From Petrov Einstein To Navier-Stokes", [arXiv:1104.5502].

[31] T. Z. Huang, Y. Ling, W. J. Pan, Y. Tian, and X. N. Wu, "From Petrov-Einstein to Navier-Stokes in spatially curved spacetime", JHEP 1110, 079,(2011) [arXiv:1107.1464].

[32] T. Z. Huang, Y. Ling, W. J. Pan, Y. Tian and X. N. Wu, "Fluid/gravity duality with Petrovlike boundary condition in a spacetime with a cosmological constant", Phys. Rev. D 85, 123531 (2012) [arXiv:1111.1576].

[33] C. Y. Zhang, Y. Ling, C. Niu, Y. Tian, X. N. Wu, "Magnetohydrodynamics from gravity", Phys. Rev. D86, 084043 (2012) [arXiv:1204.0959].

[34] X. Wu, Y. Ling, Y. Tian and C. Zhang, "Fluid/Gravity Correspondence for General Non-rotating Black Holes", Class. Quant. Grav. 30, 145012 (2013) [arXiv:1303.3736].

[35] Y. Ling, C. Niu, Y. Tian, X. N. Wu and W. Zhang, "Note on the Petrov-like boundary condition at finite cutoff surface in Gravity/Fluid duality", Phys. Rev. D 90, 043525 (2014) [arXiv:1306.5633].

[36] B. Wu, L. Zhao, "Gravity mediated holography in fluid dynamics", Nucl. Phys. B874, No.1, P177-187 (2013) [arXiv:1303.4475].

[37] B. Wu and L. Zhao, "Holographic fluid from nonminimally coupled scalar-tensor theory of gravity", Class. Quantum Grav. 31 (2014) 105018 [arXiv:1401.6487].

[38] R. G. Cai, Q. Yang and Y. L. Zhang, "Petrov type I Spacetime and Dual Relativistic Fluids", Phys. Rev. D 90, 041901 (2014) [arXiv:1401.7792].

[39] R. G. Cai, Q. Yang and Y. L. Zhang, "Petrov type I Condition and Rindler Fluid in Vacuum Einstein-Gauss-Bonnet Gravity", JHEP 1412, 147 (2014) [arXiv:1408.6488].

[40] X. Hao, B. Wu and L. Zhao, "Flat space compressible fluid as holographic dual of black hole with curved horizon", to appear in JHEP [arXiv:1412.8144].

[41] T. Moskalets and A. Nurmagambetov, "Liouville mode in Gauge/Gravity Duality", [arXiv:1409.4186]. 